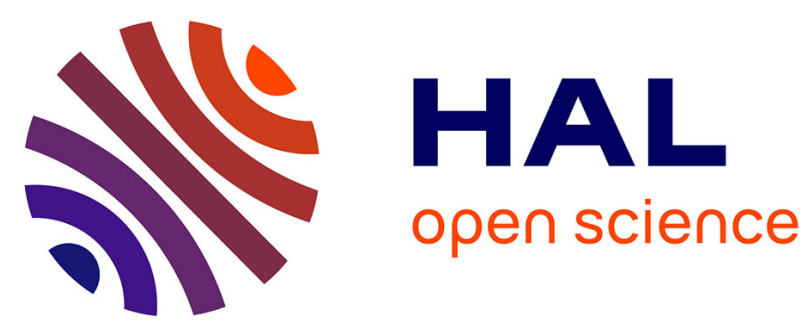

\title{
FAMOUS: a fast instrumental and computational pipeline for multiphoton microscopy applied to 3D imaging of muscle ultrastructure
}

Claire Lefort, Mathieu Chalvidal, Alexis Parenté, Véronique Blanquet, Henri Massias, Laetitia Magnol, Emilie Chouzenoux

\section{To cite this version:}

Claire Lefort, Mathieu Chalvidal, Alexis Parenté, Véronique Blanquet, Henri Massias, et al.. FAMOUS: a fast instrumental and computational pipeline for multiphoton microscopy applied to 3D imaging of muscle ultrastructure. Journal of Physics D: Applied Physics, 2021, 54 (27), pp.274005. 10.1088/1361-6463/abf8f2 . hal-03225997v2

\section{HAL Id: hal-03225997 \\ https://hal.science/hal-03225997v2}

Submitted on 15 Nov 2021

HAL is a multi-disciplinary open access archive for the deposit and dissemination of scientific research documents, whether they are published or not. The documents may come from teaching and research institutions in France or abroad, or from public or private research centers.
L'archive ouverte pluridisciplinaire HAL, est destinée au dépôt et à la diffusion de documents scientifiques de niveau recherche, publiés ou non, émanant des établissements d'enseignement et de recherche français ou étrangers, des laboratoires publics ou privés. 


\section{Abstract}

We present a new instrumental and computational pipeline named FAMOUS: Fast algorithm for 3D multiphoton microscopy of biomedical structures. This pipeline rests on a multiphoton microscopy (MPM) strategy combined with an original 3D post-processing computational approach. In the present work, FAMOUS approach is devoted to the 3D imaging of the myosin assembly of the ultrastructure of a whole striated skeletal muscle unsliced. Raw recordings of second harmonic generation (SHG) from myosin and instrumental point-spread functions (PSF) are led simultaneously all along the unsliced muscle depth. This procedure highlights a space-variant distortion of the PSF and the SHG signals and an optical degradation of the axial resolution increasing with imaging depth resulting from the optical heterogeneity of the muscle structure. A 3D mathematical modelling of the PSF, relying on the recent FIGARO method, evaluates and models the depth-variant evolution of the optical distortions. Then, the fast image deblurring algorithm BD3MG is employed to correct those non-stationary distortions all along the sample, thanks to a sounded regularized inverse problem methodology. This leads to the pipeline called FAMOUS, whose performance are highlighted for the optimization of the axial information of myosin structure, whose dimensions are close to the axial resolution limit. For the first time, the 3D organization of the myosin in skeletal muscle is visually shown from an unsliced whole muscle, starting with a solution of optical microscopy. The axial visualization of this organization presently disclosed were never shown until now without a preliminary procedure of sample slicing and labelling. Our original solution FAMOUS delivers a new point of view of this biological structure in the 3 dimensions and especially in the optical axis. Image information theoretically expected are now revealed visually in the optical axis for the first time in a whole organ unsliced and label free.

\section{Introduction}

Multiphoton microscopy (MPM) is a contactless method devoted to sample imaging of various objects, from living kingdom to materials [Larson 2011]. This attractive solution for biomedical imaging gathers several high-performance technologies into a single instrument [Zipfel 2003]. Three-dimensional (3D) imaging with a microscopic resolution [Gobel 2007] combined with the infrared range of excitation opens imaging depth at least twice as deep as one-photon microscopy [Centonze 1998]. MPM results in a powerful technique enabling biologists to observe 3D images of targets with a sub-micrometric resolution up to several hundreds of micrometers under the sample surface without any sample slicing procedure. A record of imaging depth reaching $1.6 \mathrm{~mm}$ has even been demonstrated in mouse cortex [Kobat 2011]. But deep-3D-MPM (D-3D-MPM) faces with wavefront distortions deforming visually the produced image, all along the imaging depth [Young 2010 a, Young 2010 b]. Spherical aberration results 
from refractive index mismatch which primarily degrades the focus especially in the optical axis resulting in an axially-extended point-spread-function (PSF) and limits the range of light penetration depth [Jin 2020]. Moreover, scattering results from sample heterogeneities associated to a non-uniform refractive index. These phenomena become a source of image degradation that damages visual quality of image and resolution, increasingly with imaging depth. The combination of these deleterious effects can be gathered into a "black-box of degradations" whose internal behaviour can be difficult to quantify precisely for any biological sample imaged. In that context, we can consider an overall image degradation box which can be measured and quantified numerically. Then, an adequate computational restoration strategy based on inverse problems methodology can deliver an image with attenuated visual distortions resulting in an improved visual quality.

The PSF estimation is a solution for evaluating the overall impact of the acquisition chain on the image degradation. This strategy includes the observation of reference objects through the microscope device, usually microspheres with a diameter smaller than resolution. In literature, the PSF evaluation and its evolution depending on scattering substances have been modelled with a Monte-Carlo model [Dunn 2000] and investigated experimentally in function with imaging depth [Dong 2003]. No measurable evolution of lateral PSF widths were measured in function with imaging depth, but a phenomenon of increasing the axial width with imaging depth have been highlighted and attributed to the ability to deliver sufficient ballistic photon density to the focal volume [Dunn 2000]. The impact of high numerical aperture (NA) objectives [Dong 2004] and light shaping modules based on adaptative optics [Matsumoto 2017, Doi 2018, Leemans 2020] have been also devoted to correct image deterioration associated to optical aberrations. An alternative solution consists in removing scattering constituents of the sample with optical clearing agents [Cicchi 2005], with a risk of deterioration of the biological structure.

Skeletal muscle is a specialized contractile tissue with a highly ordered structure made up of various types of fibres with different myosin heavy chain composition and metabolic states. Moreover, the muscle fibres located at the periphery of the muscle can reveal a different internal structuration compared to those located deep inside [Schiaffino 2011]. These specificities highlight an interest in analysing the muscle ultrastructure in $3 \mathrm{D}$ all along its depth and is associated to heterogeneous optical properties. The sarcomere constitutes the elementary contractile unit of muscle ultrastructure and is composed by an alternative assembly of thick filaments of myosin and thin filaments of actin [Craig 2004] with dimensions between $1.8 \mu \mathrm{m}$ and $3.0 \mu \mathrm{m}$ [Millman 1998, Hessel 2019]. When skeletal muscle samples are obtained by muscle resection and slicing, an immunofluorescence staining protocol of actin allows the localisation of actin filaments revealed by exogeneous two-photon fluorescence (TPF) imaging [Recher 2009]. The metabolic states of myofibrils and the distribution of mitochondria and NAD(P)H in skeletal muscle in vivo can also be monitored by endogenous TPF [Rothstein 2005]. But the study of the structural organisation of muscle label-free without any muscle slicing can only be followed thanks to the imaging of myosin structuration. Indeed, myosin is a protein organized with a noncentrosymmetric structure. In case of multiphoton excitation, a coherent second harmonic generation (SHG) arises label-free from domains of the sarcomere containing myosin. This property has been widely exploited in literature for revealing the 2D myosin organization [Plotnikov 2006, Lefort 2016] and detecting muscle defects [Pfeffer 2011, Raben 2013]. In both cases, 2D images of SHG from myosin are produced thanks to a slicing and labelling procedure. 3D images are then reconstructed thanks to each $2 \mathrm{D}$ serial images. 3D imaging of a muscle organisation has never been studied by SHG through a whole muscle without sample slicing and labelling. The sarcomere dimensions vary between $1.6 \mu \mathrm{m}$ and $2.5 \mu \mathrm{m}$. The radial periodical organization is thus compatible with the radial resolutions of MPM of about $0.4 \mu \mathrm{m}$, but not with the axial resolution about $1.5 \mu \mathrm{m}$ at best at sample surface. When 
imaging the axial dimension of muscle structure, the spatial organization of thick filaments of the sarcomere becomes drowned in the blur and noise of the image. For a structure as complex as a muscle, the internal optical properties are highly dependent from numerous parameters as the muscle phenotype, metabolic and lipid states. A local characterization of the image distortions is the exclusive way able to deliver true information of the structure.

The local and precise characterization of image distortions occurring non-homogeneously into a complex and thick biological structure is often necessary for an accurate restoration of images recorded deep into the sample. The compensation of image distortions amounts to solving a so-called inverse problem, with the aim of increasing the imaging depth while keeping constant visual image quality and axial information [Mertz 2009]. In literature, several strategies have been tested on biological samples with thicknesses of few tens of micrometers at best: optical strategies [Ibáñez-López 2005, Descloux 2016], computational strategies [Boutet de Monvel 2003, Dao 2015, Strohl 2015] or a combination of both [Song 2006, Doi 2018, Labouta 2012, Roider 2017].

In this publication, we propose the new instrumental and computational pipeline named FAMOUS: Fast algorithm for 3D multiphoton microscopy of biomedical structures. Starting with two preliminary steps of (1) preparation of the whole organ, combined with (2) the inclusion of microspheres, this solution provides (3) a 3D acquisition of raw images with an MPM approach, (4) a quantitative evaluation of the variations in depth of the 3D PSF, (5) a fast 3D image deblurring approach accounting for a model for depth-variant PSF, that visually improves the image quality. This solution based on 3D acquisitions delivers a new point of view and a new kind of information, here demonstrated for a muscle structure. We characterize the evolution of the PSF of the MPM device as a function of the imaging depth in two different situations. The first set of experiments involves an optical phantom constituted by a silicone matrix containing fluorescent microspheres homogeneously spread into the whole volume. This medium delivers homogeneous optical and scattering properties in the whole imaging depth that limits the wavefront distortions. Second, a mouse skeletal muscle is imaged. This living model presents a structure highly heterogeneous due to an alternative organization between two proteins, myosin and actin, resulting in a striated structure [Cooper 2000]. The image distortions resulting from both heterogeneous and homogeneous media are evaluated and compared. In each case, the PSF evolution with imaging depth is evaluated thanks to the 3D PSF fitting FIGARO algorithm [Chouzenoux 2019]. Finally, the depth-variant image distortions are numerically reduced thanks to a fast optimization-based image deblurring approach resting on a depth-variant PSF model built from the aforementioned PSF characterization step [Chalvidal 2020]. We then demonstrate that the resulting pipeline FAMOUS applied to a muscle structure improves the visual image quality especially along the muscle depth. For the first time, the fibrillary organization of myosin muscle is revealed axially in a whole organ unsliced and label-free.

\section{Materials and methods}

\subsection{Overview of the instrumental pipeline FAMOUS}

Figure 1 proposes an overview of the instrumental pipeline FAMOUS gathering 5 stages illustrated with images acquired during the procedure. The initial step consists in (1) preparing the whole organ, followed by (2) the inclusion of microspheres. Then, (3) a 3D acquisition of raw images delivers signals from PSF and biological structure simultaneously thanks to an MPM approach. Afterwards, (4) a 
quantitative evaluation of the 3D PSF and its variations all along the organ depth is led and finally, (5) a depth-variant 3D PSF model is built, and a fast 3D image deblurring approach visually improves the image quality. The first two steps are related to the biological engineering; the third one is related to microscopic acquisitions and the last two ones are related to computational engineering.

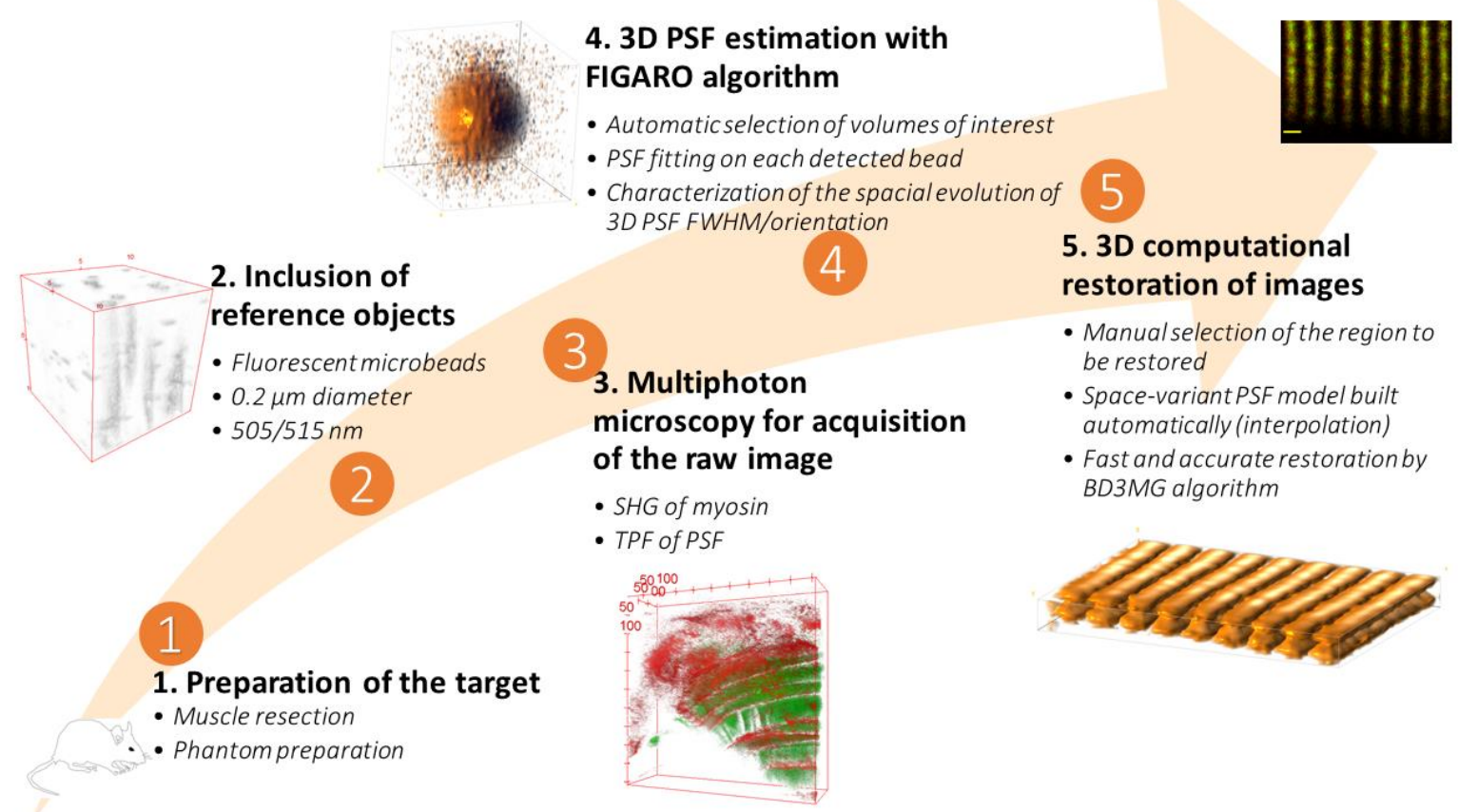

Figure 1. Overview of the instrumental pipeline FAMOUS for multiphoton muscle imaging containing five key steps, from the preparation of the target to be imaged to the production of the 3D image with an improved visual quality.

\subsection{Sample preparation and fluorescent microspheres for PSF estimations}

The impact of the imaging medium on the PSF evolution all along the imaging depth is quantified here. To that aim, the PSF is measured through MPM acquisitions of microspheres at various imaging depth for two kinds of imaging medium: a homogeneous and a heterogeneous medium.

\subsubsection{Homogeneous optical phantom including fluorescent microspheres}

The optical phantom was produced thanks to a solution of $10 \mu \mathrm{L}$ of microsphere, first mixed into $3 \mathrm{~mL}$ of polymer of Sylgard ${ }^{\circledR} 184$, a polydimethylsiloxane elastomer. The fluorescent microsphere solution was constituted by polystyrene microspheres presenting a subresolution diameter of $220 \mathrm{~nm}$ and labelled with a yellow-green fluorophore emitting a fluorescent signal at $515 \mathrm{~nm}$ (Molecular Probes ${ }^{\mathrm{TM}}$ FluoSpheres ${ }^{\mathrm{TM}}, 0.2 \mu \mathrm{m}$, yellow-green fluorescent 505/515). The two-photon absorption range was spread between 780 and $960 \mathrm{~nm}$ with a maximum absorption at $920 \mathrm{~nm}$. Their mixture with the polydimethylsiloxane elastomer resulted in a homogeneous solution. Its refractive index was measured by ellipsometry all along the phantom depth and was equal to 1.42 at $515 \mathrm{~nm}$. Then, the homogeneous mixture of polymer and microsphere was manually included into $300 \mu \mathrm{L}$ of polymerizing agent. The whole solution was then placed into a Petri dish and kept at room temperature during 24 hours. The resulting solid polymer was homogeneous optically; no de-airing was necessary. The polymerized gel has reached a total thickness of $1.5 \mathrm{~mm}$, with microsphere homogeneously spread into the whole depth. 


\subsubsection{Mouse skeletal muscle}

Extensor digitorum longus (EDL) muscles were isolated from tendon to tendon of 3-month-old wild-type FVB male mice. EDL muscles were fixed overnight at $4^{\circ} \mathrm{C}$ in $4 \%$ paraformaldehyde (PFA). Muscle tissues were washed three times in Phosphate-Buffered Saline (PBS) and incubated 3 hours at $4^{\circ} \mathrm{C}$ in solution PBS-1\% Tween20 with the yellow-green fluorescent microspheres (Molecular Probes ${ }^{\mathrm{TM}}$ FluoSpheres ${ }^{\mathrm{TM}}$, $0.2 \mu \mathrm{m}$, yellow-green fluorescent 505/515). EDL muscles were then placed into PBS. With this protocol, the fluorescent microspheres were thus distributed all inside the whole muscle and especially between the myofibrils. The microscopic acquisition of mouse muscle images was realized thanks to a water immersion objective. Thus, the EDL muscle was fixed mechanically under the microscope objective and then immersed into the PSB solution. Figure 2 illustrates in red the repartition of microspheres into the whole muscle, and in green the localisation of SHG signal from myosin of muscle.

\subsection{Multiphoton microscopy}

The method of MPM was retained thanks to its unequalled performance for high in-depth resolution compared to more standard widefield or confocal microscopy methods. Indeed, the near infrared range (NIR) of excitation involved in MPM covers a spectral window that can penetrate deeper inside living samples. Moreover, the multiphoton processes involved in MPM are associated to an inherent property of optical sectioning. In our experimental setup, the commercial multiphoton microscope from Olympus (reference BX61WI) was coupled with a tunable titanium-doped sapphire laser excitation (Ti: Sa) laser system delivering femtosecond pulses (Chameleon Ultra II, Coherent). The central wavelength of excitation was fixed at $860 \mathrm{~nm}$. A water immersion objective corrected for multiphoton acquisitions (Olympus XLPLN25XWMP, 25x, numerical aperture 1.05) focalized the beam on the sample. A 2D scanning device, composed by two galvanometric mirrors, ensured the sample scanning by the laser resulting in the production of $2 \mathrm{D}$ images of single optical plans. Scanning speed and image dimensions were fixed at $2 \mu \mathrm{s} /$ pix and 2048 pixels $\times 2048$ pixels. An axial scan allowed the translation of microscope objective, and the sequential acquisition of 2D stacks of images, spaced $2 \mu \mathrm{m}$ apart or $0.1 \mu \mathrm{m}$ apart depending on the sought information (overall or microscopic organization). The superposition of 2D stacks of images resulted in the production of 3D images of biological samples with an acquisition duration of about 10 minutes. A backward detection system was composed by a first dichroic mirror $(650 \mathrm{~nm})$ separating the excitation light from the emitted multiphoton signals. A second dichroic mirror $(470 \mathrm{~nm})$ splitted the two spectral components of multiphoton signal emitted from the sample. Each of these signals were detected by a photomultiplier tube (PMT) precede by a set of filters. The range between 495 and $540 \mathrm{~nm}$ was associated to the fluorescence of microspheres and the range between 420 and $440 \mathrm{~nm}$ corresponded to the SHG signal emitted from myosin. The acquisition procedure was managed with FluoView FV1000 software (Olympus) and produces stacks of 2D slices in Tiff format. The $2 \mathrm{D}$ image slices were then loaded with ImageJ, a freely available software dedicated to image display, edition and analysis. The 3D-plot delivered a representation of the superposition of the raw acquisitions, presented as a 3D image. Figure 2 illustrates the resulting bi-channel raw acquisition, combining SHG signal from myosin (green) and two-photon fluorescence (TPF) from microspheres (red), in the overall muscle structure. 
Figure 2. 3D representation of bi-channel raw acquisitions of the entire skeletal mouse muscle and microspheres. Extensor digitorum longus (EDL) resected from a 3 months old male wild mouse. 90 2D raw images of $2048 \times 2048$ pixels corresponding to a field of view of $500 \mu \mathrm{m} \times 500 \mu \mathrm{m}$ are recorded each $2 \mu \mathrm{m}$ along the total depth of the EDL $(180 \mu \mathrm{m})$. Green: SHG signal from myosin. Red: TPF from reference microspheres.

The resulting image represents a volume of the muscle of $500 \mu \mathrm{m} \times 500 \mu \mathrm{m} \times 100 \mu \mathrm{m}$. Such a viewing point highlights the relative location of the myofibers. For that general overview of the structure, the voxel dimension $(0.25 \mu \mathrm{m} \times 0,25 \mu \mathrm{m} \times 2 \mu \mathrm{m})$ was not a critical parameter. The recording duration was about a ten of minutes. In case of computational estimation and image recovery, the pixel dimension becomes a critical parameter for the reliable PSF estimation which is presented in next part 2.4. For an image acquisition in anticipation of the computational processing step, the voxel size becomes a crucial parameter which must be 10 times smaller than the expected resolution. In that case, a zoom on the native region is essential for the production of images with an acceptable recording duration and resulting into an image size adapted to a computational processing. The image dimension is consequently limited to a box of about $50 \mu \mathrm{m} \times 50 \mu \mathrm{m} \times 100 \mu \mathrm{m}$ at best. The time delay for such an acquisition is also about $10 \mathrm{~min}$.

\subsection{Computational approach: the pipeline FAMOUS}

\subsubsection{Overall computational strategy for improving visual quality of muscle images}

The final aim of the pipeline FAMOUS is to restore volumetric muscle data images acquired thanks to a multiphoton process in the entire depth of a whole muscle. The strategy chosen rests on the fact that a raw acquisition generates an image which can be seen as a convolution between the real signal emitted by the sample and the instrumental contribution, plus some noise. The image degradation due to optical aberrations and wavefront distortions is an overall phenomenon which can be modelled numerically by the mathematical estimation of a space-variant PSF experimentally recorded all along the organ depth. The PSF is tri-dimensional and may vary at each 3D position of the sample. In our pipeline, it was approximated by images of small enough microspheres, at various position in the 3D volume. This allowed to quantify the 3D PSF variations, in particular along the sample depth. Thanks to the simultaneous acquisition of signals from SHG in the first recording channel and microspheres (PSF signal) in the second recording channel, the signal to be restored was recorded in the one hand and the distorted PSF in the other hand. The mathematical modelling of each PSF was performed following the strategy in [Chouzenoux 2019]. This allowed to quantify and model the evolution of the PSF along the acquisition depth thus modelling the image degradation considered at each sample region as a 
phenomenon related to a combination of optical aberrations and wavefront distortions. Then, a nonblind formulation of the resulting inverse problem of image restoration was solved, by minimizing a penalized criterion thanks to an efficient and fast optimization strategy relying on the majorizationminimization principle and efficient distributed implementation on multi-cores architecture.

\subsubsection{Computational pipeline and routine for PSF estimation}

The estimation of the PSF parameters rests on the acquisition of the TPF signal emitted from microspheres, on the automatic detection for the locations of microspheres, and on the Gaussian fitting of the resulting areas led by the FIGARO algorithm. The FIGARO procedure performs the fitting of the measured PSFs with multivariate tri-dimensional Gaussian functions, relying on an original and theoretically sounded variational formulation accounting for a non-zero background, model mismatch and noisy input data [Chouzenoux 2019]. The resulting 3D PSF fitting process returns, for each region of interest containing a single microbead, a full tri-dimensional characterization (in particular, width and orientation) of the PSF. The performance of FIGARO has been previously shown in a standard twophoton fluorescence imaging setup [Chouzenoux 2019] and in a setup with a highly deteriorated excitation situation involving an ultrawide band laser light [Hortholary 2021]. In the aforementioned works, we have also quantified its superior performance, when compared to standard solutions of PSF estimation, MetroloJ and Huygens softwares, namely the most commonly used tools for this task.

Our PSF estimation pipeline was applied to the whole deep volume imaged. In this specific situation and for each of the two media (homogeneous or heterogeneous optically), an automated computational routine with MATLAB was executed. The $1^{\text {st }}$ step consisted in detecting small volumes of interests containing individual microspheres, associated with their localizations in the volume. Then, FIGARO was applied to each of the volumes. The PSF widths at the full width at half maximum (FWHM) were, in particular, returned as output of this multivariate Gaussian fitting method.

\subsubsection{Image restoration}

The last step of the pipeline FAMOUS consists in delivering the restored images. To this aim, it is necessary to have a quantitative model for the PSF variations in the whole sample. Since not all 3D positions can be covered by the microspheres, interpolation is needed to build a complete PSF map from the FIGARO results obtained at the previous step. Our experimental results suggested that PSFs mostly vary in width, and that this variation is directly linked to the position in depth of the microsphere. In contrast, their variability in orientation and with the X-Y position can be neglected. We thus built a depth-variant PSF model, where the PSF width along the 3 axis, at a given depth, is deduced from the recorded ones by bilinear interpolation. We have applied a protocol of 3D image recovery considering this depth-variant PSF model. An accelerated version of the algorithm from [Chouzenoux 2013] called the block distributed majorize-minimize memory gradient (BD3MG) is employed [Chalvidal 2020]. BDM3G minimizes a least-squares criterion, accounting for our depth-variant PSF model, regularized with a smoothed total-variation term reducing noisy artifacts and a quadratic penalty to constrain the range of the pixel intensities. This approach takes advantage of distributed asynchronous computations allowed on our multi-processors computing device. This algorithmic solution is thus ideal for solving large scale optimization problems, with a significant computational time savings with respect to the sequential i.e. non-parallelized approach from [Chouzenoux 2013], while benefiting from the same convergence guarantees. The proposed method relies on the formulation from [Cadoni 2016] that was especially developed and optimized for 3D microscopy image restoration problem involving a depthvariant blur. 


\section{Experimental results}

\subsection{PSF evolution in the depth of homogeneous and heterogeneous media}

The PSF evolution in a sample depth depends on the optical homogeneity of the sample medium. In this part, the PSF evolution into the muscle structure was compared to the PSF evolution recorded into a homogeneous optical phantom. The silicone phantom containing fluorescent microspheres was employed as a medium optically homogeneous. The skeletal mouse muscle containing the microspheres homogeneously spread into the whole structure was employed as a medium optically heterogeneous. Indeed, the alternation of proteins, metabolic states of individual myofibrils and lipid state impart to this structure non-uniform optical properties. FIGARO algorithm for PSF estimation was involved in both situations and nearly 2000 beads were identified for the homogeneous medium for a calculation duration of 72 hours and about 200 in the heterogeneous medium for a calculation time of 48 hours. The average duration of calculation was about 2 minutes per PSF in the homogeneous medium and about 15 minutes per PSF in the heterogeneous medium. The significant difference of duration for PSF fitting has resulted from the higher quantity of iterations of the FIGARO algorithm necessary for the highly deteriorated acquisition into the heterogeneous medium.

Figure $3 \mathrm{~A}$ and $3 \mathrm{~B}$ gather the PSF widths estimated by FIGARO and measured the FWHM respectively into the silicone phantom (homogeneous medium) and into the muscle sample (heterogeneous medium), in the lateral plan (XY) and in the axial plan (XZ). PSF evolution was plotted as a function of the localization of the microsphere center in the sample depth. Concerning the PSF inclination, also returned as an output of FIGARO, no trend has emerged, when correlating to the imaging depth.
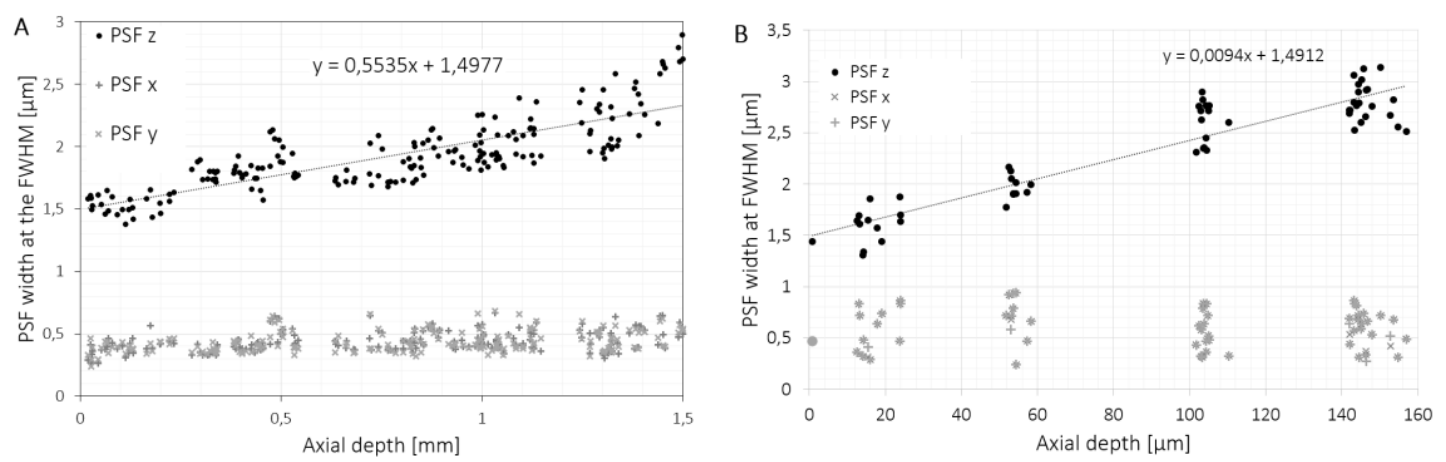

Figure 3. Evolution of axial PSF width (PSF z) and lateral PSF width (PSF $x$ and PSF y) measured at the FWHM in function with the depth in a homogeneous optical phantom of Sylgard ${ }^{\circledR} 184$ polymer $(A)$ and in a heterogeneous medium of striated skeletal muscle (B).

Figures $3 \mathrm{~A}$ and $3 \mathrm{~B}$ present the PSF evolution along the imaging depth for two situations drastically different in terms of optical homogeneity, weight and origin of wavefront distortions. We consider the optical phantom as a homogeneous reference compared to muscle structure, a highly heterogeneous structure. The question of the origin and relative repartition of image deterioration between light scattering, absorption and optical aberrations could be researched with complex setups and calculations or with a system of wavefront corrections. In the present strategy, for both media, we consider the optical distortions as an overall problem of wavefront distortions, resulting from a (non-explicitly modeled) combination between scattering, absorption and spherical aberrations. Here, the objective is to estimate numerically the image distortions resulting for the optical phenomena thanks to the PSF 
estimation for finally restore 3D images when the deterioration is complex, not fully modelled and highly space variant. We consider the image distortions and restoration problem as a whole and not as a combination of compartmentalized optical contributions to remove.

Figure 3 illustrates the drastically difference of PSF evolution for a homogeneous or a heterogeneous medium. Our results show two distinct phenomena, namely: the lateral and the axial evolutions of PSF width along imaging depth. As expected [Dunn 2000], the lateral dimension of the PSF has presented a width not affected by the imaging depth on the whole sample depth up to $1.5 \mathrm{~mm}$ or $150 \mu \mathrm{m}$, regardless of the imaging medium. The average lateral PSF width at the FWHM in the xy plan was evaluated at 0.38 $\mu \mathrm{m}$ in the homogeneous medium and at $0.43 \mu \mathrm{m}$ for the heterogeneous medium. Thus, the lateral resolution is unaffected by the wavefront distortions occurring into a homogeneous or heterogeneous medium. This corroborated an expected result: the wavefront distortions occurring during an acquisition of MPM and increasing with imaging depths has no measurable effect on lateral PSF widths.

Table 1. Axial PSF widths at FWHM at several imaging depth, extracted from experimental results.

Axial PSF width at the FWHM

\begin{tabular}{l|l|l}
\multirow{2}{*}{ Axial localization } & \multicolumn{2}{|c}{ Axial PSF width at the FWHM } \\
\cline { 2 - 3 } & Homogeneous medium & Heterogeneous medium \\
\hline Sample surface & $1.5 \mu \mathrm{m}$ & $1.5 \mu \mathrm{m}$ \\
$5 \mu \mathrm{m}$ under the sample surface & $1.5 \mu \mathrm{m}$ & $1.6 \mu \mathrm{m}$ \\
$100 \mu \mathrm{m}$ under the sample surface & $1.6 \mu \mathrm{m}$ & $2.4 \mu \mathrm{m}$ \\
$150 \mu \mathrm{m}$ under the sample surface & $1.6 \mu \mathrm{m}$ & $2.9 \mu \mathrm{m}$ \\
$0.5 \mathrm{~mm}$ under the sample surface & $1.8 \mu \mathrm{m}$ & $6.2 \mu \mathrm{m}$ (extrapolation) \\
$1 \mathrm{~mm}$ under the sample surface & $2 \mu \mathrm{m}$ & $10.9 \mu \mathrm{m}$ (extrapolation) \\
$1.5 \mathrm{~mm}$ under the sample surface & $2.4 \mu \mathrm{m}$ & $25 \mu \mathrm{m}$ (extrapolation)
\end{tabular}

Regarding the axial evolution of the PSF width at FWHM, and as expected, a dramatic effect of PSF broadening with imaging depth is highlighted in the homogeneous and heterogeneous media. In case of a homogeneous medium, the axial broadening of the PSF at the FWHM is about $3 \%$ in depth and 60 $\%$ in depth for a heterogeneous medium. Table 1 gathers some numerical examples of the axial PSF width measured at the FWHM for different imaging depth and for the homogeneous and the heterogeneous medium. An axial PSF width of $2.4 \mu \mathrm{m}$ at the FWHM is measured at an imaging depth of $1.5 \mathrm{~mm}$ under sample surface in the homogeneous medium and at an imaging depth of $100 \mu \mathrm{m}$ under sample surface for the heterogeneous medium.

These results show that the difference in the medium optical properties (refractive index mismatch and density of scattering and absorbing substances) impacts the properties of scattering, absorption and spherical aberration which directly increases the axial wavefront distortions and thus the axial PSF width. This combination impacts the density of ballistic photons delivered and then emitted from the focal volume, thus affecting the excitation and detection efficiencies in function with imaging depth [Dunn 2000, Young 2011]. This feature has a consequence on the image signal to noise ratio, degraded in function with imaging depth. The more deeply the PSF is recorded, the less the axial PSF recording is accurate. This difference directly comes out as an axial PSF broadening. In case of a D-3D-MPM experiment, the limiting factor affecting the image quality in the axial plan is the quality of signal to noise ratio.

Finally, the high difference of PSF estimation depending on imaging medium illustrated into Table 1 reveals another key point in the accuracy of the strategy chosen of MPM image post-processing. Indeed, a theoretical PSF estimation, often involved in standard image post-processing strategies, cannot deliver 
an optimal result of restauration for a deep 3D image recording. For an optimal image quality improvement, the PSF must be recorded experimentally and simultaneously directly into the imaged structure simultaneously with the sample signal to be restored. With such a solution the experimental conditions of scattering, absorption and spherical aberrations for both the signal to be restored and the PSF recording are identical. With such a protocol, image restoration can be led in optimal conditions.

\subsection{Restoration of muscle images recorded in depth considering the local PSF evolution}

The aim of the current section is to produce a 3D multiphoton image of the myosin organization of a muscle structure thanks to the recording of SHG, combined with an improved visual quality. The resulting structure of thick filaments of myosin is about $2 \mu \mathrm{m}$ long in the radial and axial directions. This dimension is compatible with the radial resolution of MPM (about $0.4 \mu \mathrm{m}$ ) but not with the axial resolution (about $1.5 \mu \mathrm{m}$ at sample surface, see Figure 3 ). In the axial direction, the periodical alignment of thick filaments of myosin is drowned into the blur and noise in the image. Here, we present the protocol of image restoration that we have implemented for muscle imaging, a highly heterogeneous structure. This strategy rests on the simultaneous acquisition of the raw SHG of myosin and the local experimental PSF associated to the mathematical fitting of its parameters.

\subsubsection{Deep-3D-MPM and deep-3D image restoration}

The myosin organization of the EDL muscle was imaged into the whole $180 \mu \mathrm{m}$ depth by SHG with our MPM system and the PSF was simultaneously recorded with the second imaging channel (Figure 2). The raw image of myosin, presented into Figure $4 \mathrm{~A}$, was extracted $100 \mu \mathrm{m}$ under the sample surface; at this imaging depth, the axial resolution was measured at $2.4 \mu \mathrm{m}$ (Table 1 ). The resulting volumetric image had the dimension of $20 \mu \mathrm{m} \times 15 \mu \mathrm{m} \times 4 \mu \mathrm{m}$. Figure $4 \mathrm{~A}$ results into the superimposition of the raw 2D stacks generating a 3D image of myosin contained into the EDL ultrastructure. Figure 4B presents the result of the 3D computational pipeline, generating an image of the periodical organization of myosin, with an augmented visual quality.
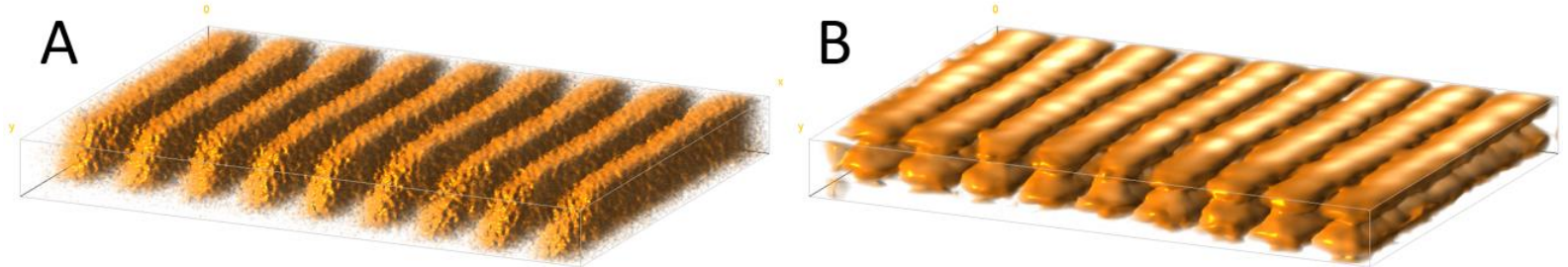

Figure 4. 3D multiphoton microscopic imaging of muscle structure recorded along a depth of $4 \mu \mathrm{m}$ before $(A)$ and after (B) image generated thanks to the pipeline FAMOUS producing a reconstruction of D-3D-MPM considering a space variant PSF.

The computational pipeline was applied for the restauration of the raw 3D image (Figure 4A). First, the PSF estimation with FIGARO is led on the signal emitted from microspheres recorded in the second channel (i.e., the sought myosin signal). The depth-variant PSF model is built, by interpolation, and the $3 D$ image recovery via BD3MG is led on the signal emitted from muscle and recorded into the first channel. The 3D reconstructed image shown in Figure 4B reveals for the first time the axial organization of thick filaments of myosin in muscle depth. Such a protocol does not require any muscle slicing of a 

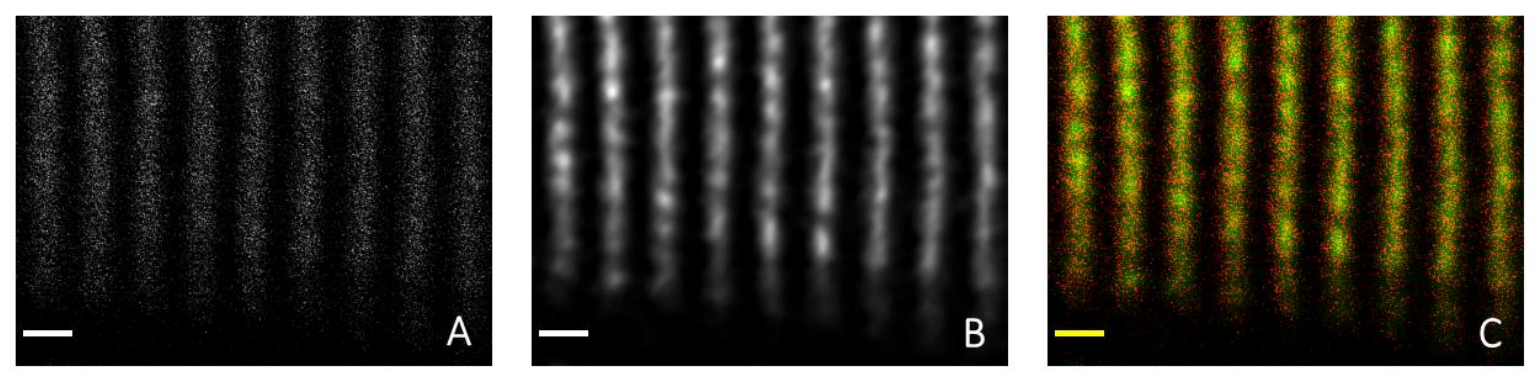

Our new instrumental strategy proposed for characterizing biological structures delivers structural information while preserving the physical integrity of the sample. Until now, such an instrumental solution did not exist with micrometre resolution deep inside the sample depth, thus revealing microscopic organization, in a whole organ not preliminarily sliced. The quantification of resulting details in the sample depth needs now a 2D analysis of images in the lateral (XY) and axial (XZ) plans.

\subsubsection{Impact of $3 \mathrm{D}$ image reconstruction on the axial resolution with the pipeline FAMOUS}

The impact of the image reconstruction is now quantitatively analysed thanks to the study of the 2D images of the EDL muscle structure in the optical plan (lateral plan, $X Y$ ) and in the muscle depth in the optical axis (axial plan, $X Z$ ). Thus, Figures 5A, 5B and 5C are related to XY plans, recorded $100 \mu \mathrm{m}$ under the sample surface. Figures 5D, 5E and 5F are related to 2D images of $X Z$ plans. Figures 5A and 5D corresponds to raw acquisitions and Figures $6 \mathrm{~B}$ and $6 \mathrm{E}$ are related to restored images. Figures $5 \mathrm{C}$ and $5 F$ superimposes raw and restored images.
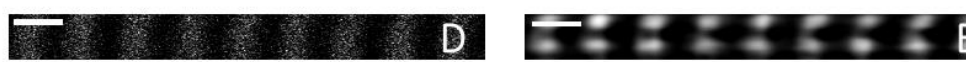

Figure 5. 2D multiphoton representations revealing myosin of muscle. $A, B$ and $C$ represents muscle image in the $X Y$ plan. D, E and $F$ represent image in the $X Z$ plan. $A$ and $D$ are raw acquisitions. $B$ and $E$ represent the images after computational retreatment considering locally the true PSF fitted experimentally. $C$ and $F$ represent the superposition of raw and retreated images respectively in the XY and XZ plan. Scale bar: $5 \mu \mathrm{m}$.

The muscle ultrastructure is composed by an assembly of sarcomeres, the basic contractile unit for striated muscle. Myosin assembly is located at the middle place of the sarcomere and generates second harmonic signal which appears thus in bright colour in multiphoton images of striated muscles. In the optical plan (Figure 5A to $5 \mathrm{C}$ ), the period of myosin, corresponding to the sarcomere period, here was equal to $2.1 \mu \mathrm{m}$ long, which greatly corresponds to the expected dimension [Millman 1998, Hessel 2019].

While the 2D representation of the myosin assembly can fairly easily reveal the sarcomere bounds in the lateral plan $(X Y)$, the question is more complex for the representation along the axial plan (XZ). Indeed, the axial resolution of MPM cannot correctly reveal the sarcomere bounds. That is why we have applied our computational pipeline to the raw 3D acquisition of muscle structure. Figure 6D to 6F illustrate the raw and visually augmented 2D axial images of myosin of EDL.

Besides, we have determined the intensity profile of the raw acquisition of SHG from myosin organization, and compared to the augmented signal resulting from the computational pipeline. Figure 
$6 \mathrm{~A}$ and $6 \mathrm{~B}$ present the normalized intensity profiles in function with acquisition positions, respectively in case of radial and axial recordings. First, the expected radial period of the sarcomere is highlighted easily, considering the raw or augmented signal (Figure 6A). The sarcomere period is revealed whatever the raw or augmented signals. However, concerning the axial period (Figure 6B), the $2 \mu \mathrm{m}$ period of the sarcomere is evidenced, for the first time, thanks to our computational pipeline.
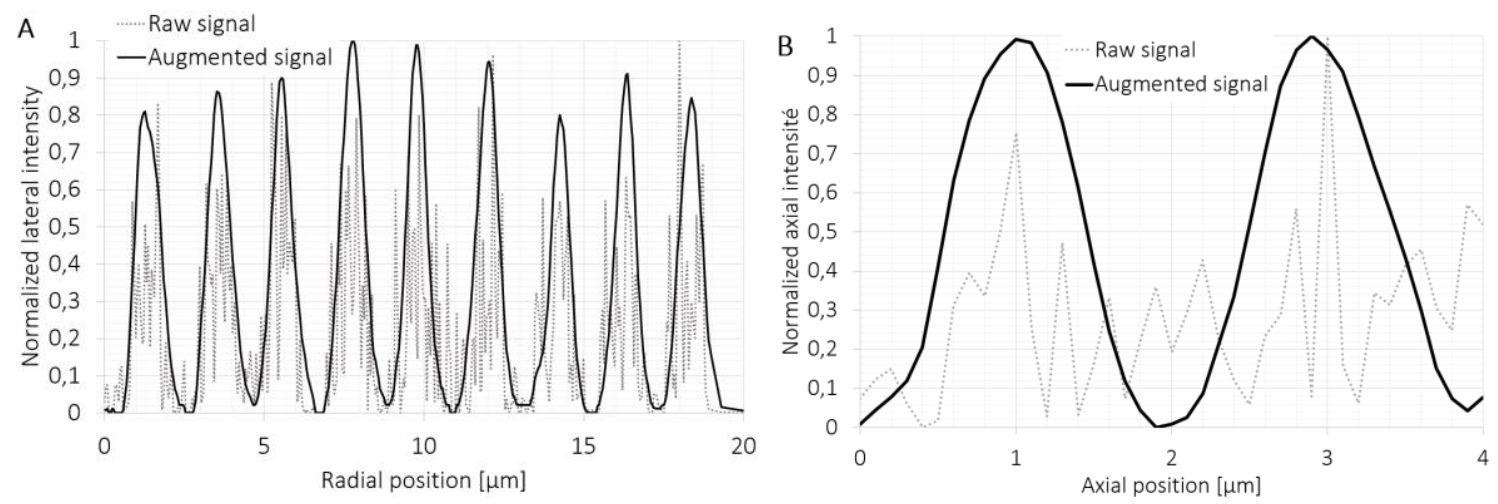

Figure 6. Normalized intensity of SHG revealing the myosin organization of muscle sarcomere. A. Raw and augmented lateral signals of SHG related to the 2D organization of myosin into the lateral plan (XY) measured from images presented in Figures 5A and 5B. B. Raw and augmented axial signals of SHG in function with imaging depth in the axial plan (XZ) measured from images presented in Figures 5D and 5E.

\subsubsection{Discussion about the axial resolution optimisation thanks to the pipeline FAMOUS}

Table 1 presents the axial resolution measured inside the muscle structure. $100 \mu \mathrm{m}$ under the muscle sample surface, the axial resolution equals $2.4 \mu \mathrm{m}$. However, the theoretical organisation of myosin has a period of about $2 \mu \mathrm{m}$. Such a level of detail in the optical axis cannot be revealed by a standard multiphoton microscope. By the way, on the raw images recorded with our standard multiphoton microscope, the expected periodicity of myosin of the $2 \mu \mathrm{m}$ is drowned into blur and noise (Figures 4A, $5 \mathrm{D}$ and $6 \mathrm{~B})$. Starting from these raw acquisitions of muscle signals combined with the PSF recordings and estimations, the pipeline FAMOUS has delivered images showing the visualization of the expected periodicity of myosin in the depth axis of the muscle. Figures $4 B, 5 E$ and $6 B$ are revealing the axial amelioration of resolution thus identifying the expected $2 \mu \mathrm{m}$ of myosin periodicity. The expected periodical structure of myosin, visible with an optimized visual quality is now possible to observe in the axial direction and in the depth of a whole muscle. It is the first time that the axial structuration of myosin is revealed by an optical solution of microscopy. The pipeline FAMOUS has thus delivered a significant axial resolution improvement.

\section{Conclusion}

Optical distortions occurring during microscopic acquisitions for skeletal muscle display a heterogeneous variability which depends on many parameters related to the sample imaged and to the instrumental setup: metabolic state, lipid, protein densities and imaging depth for example. Optical distortions in skeletal striated muscle would vary from one sample to another making unsuitable the generalization of the optical distortions occurring in the related organ. Currently, the solution involved for producing 3D views of biological samples with a microscopic level of details is mainly electron cryotomography where sample is initially fixed and dehydrated or frozen. 2D slices are then produced and imaged with a transmission electron microscope. The 2D images from individual slices are then 
superimposed and a 3D image is reconstructed. But, as far as the sample slicing is an indispensable step, the structural integrity of the sample is necessarily altered. Details of the structure can be lost due to the physical slicing procedure.

Optical solutions constitute the exclusive methods able to image biological sample with a soft and contactless protocol, associated with a micrometric resolution. Besides, the multiphoton process delivers an optical sectioning ability in the infrared range compatible with deep and 3D imaging without any physical slicing. Moreover, in some cases with a multiphoton excitation, the structure imaged can generate an optical signal label-free; for example, the structural organization of myosin generates a second harmonic signal due to its noncentrosymmetric organization. But the axial perturbation of the excitation photons due to scattering or absorption phenomena, deteriorates drastically the image quality and signal to noise ratio, especially when the acquisition is lead into a heterogeneous medium, several tens of micrometres under the sample surface. Computational strategies, shrewdly adjusted to each biological sample individually imaged, allow to consider the distortion phenomenon individually for each situation as an overall problem without paying attention to their origin.

In this publication, we have developed an instrumental and computational pipeline FAMOUS devoted to 3D muscle ultrastructure imaging. This image acquisition and restoration pipeline combines adequately multiphoton microscopy acquisition and a computational post-processing strategy. By performing the PSF estimation in each local position of the sample and fitting mathematically its physical parameters, we have obtained an accurate model of the true experimental complex PSF. This led us to an accurate image restoration, thanks to an inverse problem-based computational strategy. We have thus highlighted here that a measured PSF, located at the exact place of the image volume of interest, and described with an accurate mathematical representation, is of first importance for PSF variations modelling and thus for a relevant image reconstruction. This property is especially true in case of a 3D image reconstruction in the depth of a whole organ as a muscle, and for reaching an assessed visual quality. Thanks to this accurate strategy, we have shown that muscle structure, a highly heterogeneous edifice, can reveal the sarcomere organization in the 3 dimensions with a periodical length of about 2 $\mu \mathrm{m}$ in the radial plan as expected. For the first time, thanks to our instrumental pipeline combined with a specific computational strategy, we have shown that this periodical structure of sarcomere can also be highlighted in the axial direction, thus revealing a significant axial resolution optimization. This image reconstruction strategy results from a post-processing strategy, and image information theoretically expected are now revealed for the first time. The axial visualization of the muscle organization presently disclosed were never shown until now due to the lacks of adapted instrumental solution.

\section{Acknowledgments}

This work was supported by the European Research Council Starting Grant MAJORIS ERC-2019-STG-850925. We especially thank Jean-Christophe Orlianges for the measurement of refractive index of Sylgard phantom with ellipsometry. We would also like to thank Jean-Christophe Pesquet (CentraleSupélec, France) for fruitful discussions and insightful comments. 
[Boutet de Monvel 2003] J. Boutet de Monvel, E. Scarfone, S. Le Calvez, M. Ulfendahl, "Image-Adaptive Deconvolution for Three-Dimensional Deep Biological Imaging", Biophysical Journal, 85, 3991-4001 (2003)

[Cadoni 2016] S. Cadoni, E. Chouzenoux, J.-C. Pesquet, C. Chaux, "A block parallel majorize-minimize memory gradient algorithm", Proceedings - International Conference on Image Processing, 7532949, 3194-3198 (2016)

[Centonze 1998] V. E. Centonze, J. G. White, "Multiphoton excitation provides optical sections from deeper within scattering specimens than confocal imaging", Biophysical Journal, 75 (4), 2015-2024 (1998)

[Chalvidal 2020] M. Chalvidal and E. Chouzenoux, "Block Distributed 3MG Algorithm and its Application to 3D Image Restoration", IEEE International Conference on Image Processing (ICIP 2020), Virtual Conference (2020)

[Chouzenoux 2013] E. Chouzenoux, A. Jezierska, J.-C. Pesquet and H. Talbot, "A Majorize-Minimize Subspace Approach for I2-I0 Image Regularization", SIAM Journal on Imaging Science, 6 (1) 563-591 (2013)

[Cooper 2000] GM Cooper, "The Cell: A Molecular Approach. 2nd edition", Sunderland (MA), Sinauer Associates (2000)

[Chouzenoux 2019] E. Chouzenoux, T. T. K. Lau, C. Lefort, J.-C. Pesquet, "Optimal multivariate gaussian fitting with applications to PSF modeling in two-photon microscopy imaging", Journal of Mathematical Imaging and Vision 61 (7), 1037-1050 (2019)

[Craig 2004] R. Craig, R. Padron, "Muscle contraction - Molecular Structure of the sarcomere”, Myology, pp.129166, Edition: 3rd edition, ISBN 0-07-137180-x, The McGraw-Hill Companies, Inc. (2004)

[Dao 2015] L. Dao, B. Glancy, B. Lucotte, L-C. Chang, R. S. Balaban, L-Y. Hsu, “A Model-based approach for microvasculature structure distortion correction in two-photon fluorescence microscopy images",

[Descloux 2016] A. Descloux, L. V. Amitonova, P. W. H. Pinkse, "Aberrations of the point spread function of a multimode fiber due to partial mode excitation", Optics Express, 24 (16), 18501-18512 (2016)

[Diaspro 2002] A. Diaspro, "Confocal and Two-Photon Microscopy: Foundations, Applications and Advances", pp 576, Wiley-Blackwell (2002)

[Doi 2018], A. Doi, R. Oketani, Y. Nawa, K. Fujita, "High-resolution imaging in two-photon excitation microscopy using in situ estimations of the point spread function", Biomedical Optics Express, 9 (1), 202-213 (2018)

[Dong 2004] C-Y Dong, B. Yu, P. D. Kaplan, P. T. C. So, "Performances of high numerical aperture water and oil immersion objective in deep-tissue, multiphoton microscopic imaging of excised human skin", Microscopy Research and Technique, 63, 81-86 (2004)

[Dunn 2000] Andrew K. Dunn, Vincent P. Wallace, Mariah Coleno, Michael W. Berns, and Bruce J. Tromberg, "Influence of optical properties on two-photon fluorescence imaging in turbid samples", Applied Optics, 39 (7), 1194-1201 (2000)

[Gobel 2007] W. Gobel, B. M. Kampa, F. Helmchen, "Imaging cellular network dynamics in three dimensions using fast 3D laser scanning", Nature Methods, 4 (1), 73-79 (2007)

[Hortholary 2021] T. Hortholary, C. Carrion, E. Chouzenoux, J.-C. Pesquet, C. Lefort, "Multiplex-multiphoton microscopy and computational strategy for biomedical imaging", Microscopy Research and Technique, in press (2021)

[lbáñez-López 2005] C. Ibáñez-López, G. Saavedra, K. Plamann, G. Boyer, M. Martínez-Corral, “Quasi-spherical focal spot in two-photon scanning microscopy by three-ring apodization", Microscopy Research and Technique, 67 (1), 22-26 (2005)

[Hessel 2019] A. L. Hessel, V. Joumaa, S. Eck, W. Herzog, K. C. Nishikawa, "Optimal length, calcium sensitivity and twitch characteristics of skeletal muscles from mdm mice with a deletion in N2A titin", Journal of Experimental Biology 222 (2019)

[Jin 2020] C. Jin, L. Kong, H. Dana, H. Xie, L. Cao, G. Jin, Q. Dai, “Advances in point spread function engineering for functional imaging of neural circuits in vivo", J. Phys. D: Appl. Phys. 53 (38), 383001 (2020)

[Kobat 2011] D. Kobat, N. G. Horton, C. Xu, "In vivo two-photon microscopy to 1.6-mm depth in mouse cortex", Journal of Biomedical Optics 16 (10), 106014 (2011)

[Labouta 2012] H. I. Labouta, M. Hampel, S. Thude, K. Reutlinger, K.-H. Kostka, M. Schneider, "Depth profiling of gold nanoparticles and characterization of point spread functions in reconstructed and human skin using multiphoton microscopy", Journal of Biophotonics, 5 (1), 85-96 (2012)

[Larson 2011] A. M. Larson, “Multiphoton microscopy', Nature Photonics, 5, 1 (2011) 
[Leemans 2020] S. Leemans, A. Dvornikov, T. Gallagher, E. Gratton, "AO DIVER: Development of a threedimensional adaptive optics system to advance the depth limits of multiphoton imaging", APL Photonics, 5, 120801 (2020)

[Lefort 2016] C. Lefort, R. P O'Connor, V. Blanquet, L. Magnol, H. Kano, V. Tombelaine, P. Lévêque, V. Couderc, P. Leproux, "Multicolor multiphoton microscopy based on a nanosecond supercontinuum laser source", Journal of Biophotonics, 9 (7), 709-714 (2016)

[Mertz 2009] J. Mertz, "Introduction to Optical Microscopy", Roberts \& Company Publishers (2009)

[Millman 1998] B. M. Millman, "The Filament Lattice of Striated Muscle", Physiological Reviews, 78 (2), 359-391 (1998)

[Pfeffer 2011] C. P. Pfeffer, B. R. Olsen, F. Ganikhanov, F. Légaré, "Imaging skeletal muscle using second harmonic generation and coherent anti-Stokes Raman scattering microscopy", Biomedical Optics Express, 2 (5), 1366 - 1376 (2011)

[Plotnikov 2006] S. V. Plotnikov, A. C. Millard, P. J. Campagnola, W. A. Mohler, "Characterization of the MyosinBased Source for Second-Harmonic Generation from Muscle Sarcomeres", Biophys J., 90 (2), 693-703 (2006)

[Raben 2013] W. Liu, N. Raben, E. Ralston, "Quantitative evaluation of skeletal muscle defects in second harmonic generation images", Journal of Biomedical Optics, 18 (2), 26005 (2013)

[Recher 2009] G. Recher, D. Rouède, P. Richard, A. Simon, J.-J. Bellanger, F. Tiaho, "Three distinct sarcomeric patterns of skeletal muscle revealed by SHG and TPEF Microscopy", Optics Express, 17 (22) 19763-19777 (2009)

[Roider 2017] C. Roider, R. Piestun, A. Jesacher, "3D image scanning microscopy with engineered excitation and detection", Optica, 4 (11), 1373-1381 (2017)

[Rothstein 2005] E. C. Rothstein, S. Carroll, C. A. Combs, P. D. Jobsis, R. S. Balaban, "Skeletal Muscle NAD(P)H TwoPhoton Fluorescence Microscopy In Vivo: Topology and Optical Inner Filters", Biophysical Journal, 88 (3) 2165 2176 (2005)

[Schiaffino 2011] S. Schiaffino, C. Reggiani, "Fiber types in mammalian skeletal muscles", Physiol Rev, 91 (4), 1447-1531 (2011)

[Song 2006] W. Song, J. Lee, H-S Kwon, "Enhancement of imaging depth of two-photon microscopy using pinholes: Analytical simulation and experiments", Optics Express, 20 (8), 20605-20622 (2012)

[Strohl 2015] F. Strohl, C. F. Kaminski, "A joint Richardson - Lucy deconvolution algorithm for the reconstruction of multifocal structured illumination microscopy data", Methods Appl. Fluroesc, 3, 014002 (2015)

[Young 2010 a] P. A. Young, S. G. Clendenon, J. M. Byars, R. S. Decca, K. W. Dunn, "The effects of spherical aberration on multiphoton fluorescence excitation microscopy", Journal of Microscopy, 242 (2), 157-165 (2010) [Young 2010 b] P. A. Young, S. G. Clendenon, J. M. Byars, K. W. Dunn, "The effects of refractive index heterogeneity within kidney tissue on multiphoton fluorescence excitation microscopy", Journal of Microscopy, 242 (2), 148-156 (2010)

[Zipfel 2003] W. R. Zipfel, R.M. Williams, W. W. Webb, "Nonlinear magic: Multiphoton microscopy in the biosciences", Nature Biotechnology, 21 (11), 1369-1377 